\title{
Entre deux mondes : la recherche et les associations en éducation à l'environnement
}

Jean-Étienne Bidou

\section{(2) OpenEdition}

\section{Journals}

Édition électronique

URL : http://journals.openedition.org/ere/616

DOI : $10.4000 /$ ere.616

ISSN : 2561-2271

Éditeur

Centr'ERE

Édition imprimée

Date de publication : 1 septembre 2016

ISSN : 1373-9689

Référence électronique

Jean-Étienne Bidou, «Entre deux mondes : la recherche et les associations en éducation à

l'environnement », Éducation relative à l'environnement [En ligne], Volume 13 - 1 | 2016, mis en ligne le 15 septembre 2016, consulté le 24 septembre 2020. URL : http://journals.openedition.org/ere/616 ; DOI : https://doi.org/10.4000/ere.616 


\title{
Entre deux mondes : la recherche et les associations en éducation à l'environnement
}

\author{
Jean-Étienne Bidou
}

1 Cette dernière décennie a vu un accroissement considérable de la capacité des associations à produire de la connaissance. En témoigne leur présence de plus en plus grande dans le domaine de l'expertise, de la consultation, de l'animation des conférences de citoyens. Déjà, le terme de Tiers secteur de la connaissance et de l'innovation a été forgé pour rendre compte de l'émergence d'une recherche coopérative, démocratique dans une démarche d'appropriation sociale des connaissances. Dans ce régime d'expérimentation collective, l'action des associations a toute sa place.

2 Il s'agit là d'une altération importante au modèle occidental classique fondé sur une séparation entre la connaissance et l'action, le domaine du savant et celui de l'homme du commun, y compris le citoyen participant à la vie de la Cité. Ce cloisonnement est de plus en plus contesté depuis un demi-siècle, en fait, depuis que notre société est conçue comme une société du risque.

\section{Vers une communauté étendue de pairs ?}

3 Pour la science post-normale (Funtowicz et Ravetz, 1991), dès lors que l'incertitude est grande et/ou les enjeux sont importants, la résolution des problèmes doit s'adresser à une "communauté étendue de pairs" qui apportent leurs savoirs, qu'ils soient scientifiques ou profanes. Ces pairs sont ceux qui vont être affectés par la décision et qui sont préparés à entrer dans la discussion. Les faits étendus (extended facts), qui incluent les savoirs locaux, peuvent être utilisés dans la discussion.

4 La question de l'incertitude est aussi au centre de la réflexion de Callon, Lascoumes et Barthes (2001) qui estiment que la double délégation, celle du citoyen au profit des élus dans la sphère politique et celle du profane en direction des chercheurs professionnels 
dans le domaine de la production des connaissances et des innovations techniques, ne suffit plus pour appréhender les enjeux contemporains. Nous sommes dans une situation où le citoyen en tant que dépositaire d'une partie de la souveraineté collective générale et le profane en tant qu'utilisateur possible d'innovations ou de services doivent s'approprier cette capacité de produire des connaissances ; ils doivent pouvoir infléchir le cours de leur production et participer à des choix concernant l'organisation de la vie collective. Supprimer ces cloisonnements suppose la mise en œuvre de ce que Callon et coll. (2001) appellent des forums hybrides.

De fait, depuis quelques décennies, la société civile s'est dotée de capacités d'expertise dans le champ des grands risques ou encore de la médecine, qui lui permettent d'intervenir dans les démarches de recherche et d'influencer le débat public. Cela est vrai aussi en Éducation à l'environnement qui revendique depuis l'origine un dialogue entre chercheurs et praticiens de l'éducation formelle et non formelle.

6 Ces changements épistémologiques confortent la position que l'Éducation populaire avait prise bien antérieurement, mais pour des raisons philosophiques. Pour Richez (2006), si l'éducation populaire bénéficie aujourd'hui d'un regain d'intérêt, celui-ci est lié à des causes conjoncturelles telles les crises de l'école, de l'action culturelle, de la politique de la ville et des institutions du temps libre, mais aussi à un changement structurel qui est justement celui de "nouveaux rapports au savoir à travers le développement de la société de la connaissance et de l'information et de nouvelles formes de lien social qui s'expriment tant à travers de nouvelles modalités d'engagement que de l'explosion du phénomène associatif ».

Cependant, à travers les projets de recherche, colloques et séminaires qui se succèdent, l'expérience montre que les échanges peinent à se mettre en place. Du point de vue des associations, des questions fondamentales restent grande ouvertes : quels buts assigne$\mathrm{t}$-on à la recherche ? Comment dans un milieu associatif peut-on tenter de mettre en synergie formation et recherche, avec ses espérances et ses limites? Quelles relations peut-on établir avec la recherche académique?

\section{Quelle économie de la connaissance?}

Malgré les injonctions à établir un nouveau régime d'innovation où collaboreraient les organismes de recherche et de formation, de toutes origines, et la société civile, la nouvelle économie de la connaissance peine à s'extraire du fonctionnement économique général qui tend toujours à concentrer les acteurs dans leur spécialité. La collaboration entre la recherche institutionnelle et les associations n'a rien d'évident.

9 Le monde associatif est en expansion depuis plusieurs décennies. Les créations dépassent largement les dissolutions et ce mouvement créée des tensions, à la fois sur le travail bénévole qui n'est pas extensible, et sur les subventions publiques surtout, mais aussi privées. La concurrence est donc vive, y compris dans le secteur environnemental avec pour conséquences une situation précaire des petites associations. C'est chez elles que depuis 2010, les difficultés se sont traduites par des pertes d'emploi salarié. On comprend que dans ce contexte, l'activité de recherche ne fasse pas partie des priorités des associations environnementales ou qui travaillent dans le secteur de l'Éducation à l'environnement et au développement durable (EEDD), sauf, s'il s'agit d'activités financées et qui font partie du projet de l'association. 
10 De son côté, l'entrée du monde universitaire dans l'économie de la connaissance s'est traduite par le renforcement de l'évaluation de ses résultats. La diffusion des résultats scientifiques avait pour but initial de communiquer de nouvelles informations, données, théories afin de créer un dialogue international à travers lequel chaque auteur participerait à l'avancée de la recherche. Désormais, cette diffusion est aussi la base de tentatives de plus en plus nombreuses de décrire l'accumulation de savoirs comme quelque chose de quantifiable et que d'ailleurs, les méthodes d'évaluation de la recherche tentent de quantifier. Les publications des chercheurs, les brevets, les contrats sont considérés comme les meilleurs indicateurs de production, et certainement les plus faciles à mettre en œuvre.

11 Les chercheurs sont classés en "publiant» et «non-publiant» sur la base de procédures bibliométriques informatisées utilisées pour évaluer les travaux des chercheurs : nombre de publications dans les revues, si possible anglophones, à impact factor élevé et nombre de citations de ses articles par d'autres auteurs (H index), qui peuvent être synthétisés suivant des critères variés. Il semble normal que la publication d'un article présentant des résultats de qualité entre en compte dans l'évaluation des activités de recherche, mais ce système influence désormais le rapport des chercheurs vis-à-vis de la publication et engendre des dérives individuelles et collectives: multiplication des publications d'un intérêt limité, tronçonnement des résultats, autocitations, ou citations alternes qui traduisent les manipulations du système. Dans un sens plus large, il induit l'uniformisation des pratiques scientifiques et la compétition généralisée entre individus, laboratoires, et revues scientifiques. On comprend dès lors qu'un chercheur, à moins qu'il n'ait fait de la recherche- action ou de l'hybridation des savoirs, sa spécialité a finalement assez peu d'intérêt à entrer dans ce domaine d'activité.

12 Voilà donc deux mondes, celui des associations et le monde universitaire, que la nouvelle société de la connaissance devrait engager dans une collaboration toujours plus étroite mais qui sont recentrés sur le cœur de leur métier en raison de leur mode de fonctionnement économique. En première analyse, la spécialisation croissante des activités engendrée par une recherche d'efficacité milite contre les initiatives transversales, les incursions hors du domaine académique. Mais à cette difficulté s'ajoute l'opposition fondamentale entre théorie et pratique qui envahit tout travail collaboratif.

\section{L'académie et la division du travail intellectuel}

Il ne suffit pas de convier praticiens et chercheurs à mêmes évènements ou à les rassembler dans des projets de recherche communs. Il faut aussi construire les méthodes. C'est sur ce point que les difficultés sont particulièrement nombreuses et les résistances individuelles et institutionnelles fortes. Mais plus encore, le clivage extrêmement profond qui sépare à la fois, dans leur nature et aussi dans les pratiques, savoirs savants et savoirs profanes, traverse à différentes échelles les programmes partenariaux, les recherches actions et jusqu'aux travaux de co-écriture. Ce constat n'est pas spécifique à l'éducation à l'environnement. Depuis longtemps, les chercheurs travaillant dans l'éducation formelle, les sciences de la santé, l'économie sociale ou la gestion ont fait ce même constat et certains ont même pu nier la possibilité de concilier scientificité et pertinence pratique. 
14 Peu d'évaluations sont faites de la recherche partenariale entre universités et associations. Pourtant, comme le signale Dominique Cottereau (article de ce numéro), un certain nombre de programmes existent en France, tels les PICRI (Partenariats Institutions Citoyens) ou les ASOSC (Appropriation Sociale des Sciences), généralement dérivées des Alliances de recherche universités-communautés (ARUC) québécois qui fonctionnent depuis plus d'une dizaine d'années. Il semble cependant que l'amalgame soit faible. Passées les premières opérations, celles de la définition commune du projet après un diagnostic partagé, chacun revient à ses fonctions : la collecte des données et la production des connaissances aux chercheurs, la dissémination des résultats et leur transfert aux acteurs de terrain.

15 C'est un problème général à la plupart des recherches-actions. Dans cette démarche qui naissait à la fois d'une volonté de changement et d'une intention de recherche, il est difficile, au-delà de la définition conjointe du problème à résoudre, de maintenir dans la durée l'équilibre entre les buts des acteurs et des chercheurs, l'équivalence de leurs savoirs, un contrôle partagé de la démarche. La position du chercheur reste ambiguë : médiateur, animateur, facilitateur ou même simple individu, elle pose la question la question de l'égalité dans le groupe, et du contrôle de la recherche-action.

Le problème de la division des tâches se pose au même titre à l'échelle élémentaire, celle de la co-écriture d'articles entre chercheurs académiques et acteurs de terrain. Lorsqu'ils prennent en charge l'écriture, les acteurs de terrain fournissent l'expérience et son récit. Le rôle du chercheur est l'adaptation au contexte académique c'est-à-dire la problématisation et la discussion de l'expérience. Les références bibliographiques, les arguments pré-construits qui émaillent la discussion sont une façon de la raccorder au tissu de connaissances académiques et ainsi d'établir l'accréditation des auteurs auprès de la communauté scientifique qui va valider, ou non, leur travail.

17 Notons seulement que la division du travail institutionnalisée entre associations, qui animent sur le terrain et qui ont des questions, et les chercheurs qui mettent en place les protocoles de recherche, constitue un clivage fondamental, très difficile à résorber.

\section{Que faire?}

18 A côté de cette voie de la collaboration avec le monde académique, voie malaisée et qui ne produit pas forcément les résultats escomptés, les associations disposent d'une alternative, celle de se cantonner à une recherche autonome. Il s'agit là d'une tendance forte qui se trouve au fondement idéologique de l'Éducation Populaire. Celle-ci considère que tous sont habilités à réfléchir à leurs propres problèmes et à produire de la connaissance pour l'action. Chacun pouvant participer au développement de l'intelligence collective, il n'y a aucune raison de laisser la recherche pédagogique aux mains de chercheurs académiques, fussent-ils engagés dans la recherche de terrain. C'est depuis longtemps la position de l'École Moderne (Institut Coopératif de l'École Moderne ou mouvement Freinet). Peyronie (2013) rappelle que le fonctionnement du mouvement Freinet en intellectuel collectif assure, en effet, une recherche pédagogique autonome depuis plus d'un demi-siècle. Elle est le produit des enseignants ou des animateurs eux-mêmes dans leur fonction de "praticien-chercheur ». La contrepartie de cette autonomie est évidemment la faiblesse des liens avec la recherche académique bien que, depuis quelques décennies, les relations soient plus positives. 
19 Dans certains contextes, des outils sont particulièrement adaptés, telle la rechercheaction participative. $\mathrm{Si}$, comme toutes les recherches-actions, elle a pour but un changement social, les sujets participants sont co-chercheurs, gardent le contrôle de la recherche dans un processus qui est autant recherche, appropriation de connaissances que renforcement du pouvoir d'agir. Mais dans l'idée de Paulo Freire qui en est à l'origine, il s'agissait aussi d'un affranchissement par rapport à la recherche conventionnelle soupçonnée de véhiculer les intérêts économiques dominants (Freire, 1982). D'autres pistes ont été ouvertes dans les années 90, dans le domaine de la praxéologie à la suite de la diffusion de l'ouvrage de Schön, qui a donné des outils et l'ambition de réfléchir à l'agir professionnel. Il existe donc un outillage conceptuel tout à fait suffisant pour une recherche autonome. Se pose alors le problème de la validation des résultats par la communauté académique et de leur diffusion dans ces arènes ; mais, issus d'une recherche locale, ils n'ont pas forcément vocation à cela.

20 C'est une telle recherche-action que Dominique Cottereau décrit et analyse. Elle est menée par quatre associations bretonnes autour de la mise en œuvre démarches pédagogiques innovantes et de leur efficacité : quatre parcours, aux contextes et aux questions de recherches différentes. Si elle débouche après une année de rencontres et de travail sur un approfondissement des savoirs et des savoir-faire et comme souvent dans les recherches-actions avec des équipes lancées dans des boucles d'apprentissage, la question se pose de la validité scientifique de ces savoirs. Elaborés hors du contexte de collaboration avec la recherche universitaire, non validés par celle-ci, il s'agit de savoirs intermédiaires, entre le savoir personnel et le savoir scientifique, mais ils sont néanmoins généralisables et socialisables, en capacité d'être transmis.

21 C'est aussi le continuum entre la position de recherche et la recherche scientifique qui est interrogé dans l'article de Annie Bauer et Jean_Étienne Bidou. Conçus comme un recueil de pratiques, les livrets de l'Ifrée ont pour but, sur des sujets spécifiques qui intéressent les praticiens de l'Éducation à l'environnement, de mettre à leur disposition des ressources pour les aider à se lancer dans un projet. En explorant un champ, sélectionnant les pratiques, proposant des grilles de lecture, ils comportent des éléments de formalisation qui en font, dans une certaine mesure, un travail de recherche. Mais ils n'en ont ni la rigueur, ni la relation avec un cadre théorique que demanderait une analyse praxéologique aboutie. Ce n'est pas leur but. C'est ainsi le cas d'un très grand nombre de travaux entrepris comme une résolution de problème local ou un réseau d'échange de pratiques et qui pourraient soutenir assez aisément une recherche académique.

22 C'est le pas qui a été franchi par deux expériences de recherche, toutes deux dans le cadre d'un doctorat. Caroline Leininger-Frézal revient sur les circonstances de sa recherche basée sur une enquête sur les pratiques enseignantes en EEDD et développée en collaboration avec le Groupe Régional d'Animation et d'Initiation à la Nature et à l'Environnement ( GRAINE) de la région Rhône-Alpes. Co-construction du dispositif de recherche dans un lieu que l'auteure, en s'appuyant sur la sociologie de la traduction, apparente à un laboratoire éphémère, lieu d'un collectif de recherche.

23 La collaboration entre un chercheur et une équipe d'animateurs sur un projet pose à la fois des problèmes de positionnement dans et hors de l'équipe, mais aussi du rôle du chercheur dans l'action. C'est cette interaction complexe que démêlent Laura Seguin et Raoul Girand à propos d'une conférence de citoyens sur l'eau. Elle montre que c'est finalement le corpus de sa recherche plus que sa propre analyse qui est source de 
réflexion pour les animateurs, et les conduit à repenser leur pratique d'un point de vue critique. Ainsi, il s'agit moins de traduction entre deux mondes que d'un aménagement de l'espace intermédiaire entre connaissance et action, où se déroule la co-construction de la recherche commune (Lyet, 2011).

Cet espace de transaction peut être organisé grâce à l'emploi des technologies de l'information et de la communication. Le projet présenté par Jean-Marc Douguet et coll. a pour but de faciliter la discussion entre parties prenantes sur les questions de justice environnementale. La justice environnementale, notion construite par les mouvements sociaux dans leur lutte contre les inégalités se prête à l'hybridation entre les discours associatifs et expert. L'outil informatique peut aider à mobiliser les différents acteurs et leurs connaissances pour délibérer autour des enjeux environnementaux et des modes de gouvernance plus justes.

Un aspect qui a beaucoup été travaillé dans ce numéro est celui de la transférabilité des résultats de recherches-actions. Cet objectif est au cœur de l'article de Christine Vergnolle Mainar Comment, à partir de recherches collaboratives forcément très contextualisées (ici, des actions d'EEDD dans des écoles primaires d'une vallée pyrénéenne et à propos du futur de cette vallée, associant enseignants, chercheurs en éducation et en sciences humaines) aboutir à une ingénierie éducative sinon reproduisible, du moins transférable à d'autres territoires. Ceci passe par la modélisation non de l'évolution du territoire, ni des outils pédagogiques mis en œuvre, mais de la démarche "d'arpentage du temps et de projection vers le(s) futur(s) et qui semble applicable à d'autres territoires.

Donner à nouveau du sens aux savoirs, scolaires ou autres, demande à organiser l'échange et le partage des connaissances. Pour Marylin Lair, qui étudie la formation entre pairs chez les animateurs d'associations d'éducation à l'environnement, l'interaction développe non seulement des compétences nouvelles mais aussi favorise la construction d'une identité professionnelle. Que cette formation entre pairs puisse s'organiser dans un réseau d'éducation à l'environnement montre bien l'influence qu'ont pu avoir les idées d'Ivan Illitch sur l'intérêt des dynamiques coopératives dans l'élaboration et l'appropriation des savoirs, en marge des institutions.

L'institution scolaire, c'est pourtant le domaine qui a été investi par le mouvement Établissements verts Brundtland (EVB-CSQ). Initié juste après le Sommet de la Terre de Rio par la Centrale de l'Enseignement du Québec, porté donc par le secteur syndical, ce mouvement regroupe à l'heure actuelle plus de 1400 établissements. Jean Robitaille retrace l'histoire des EVB et identifie des pistes de recherche à développer en termes de travail sur les archives, sur l'évaluation des effets éducatifs, sur la transformation des structures et également sur la prospective : autant de questions qui apparaissent dans un projet de recherche partenariale que le mouvement a initié avec l'Université du Québec à Montréal.

28 À travers l'ensemble de ces articles, on peut donc finalement bien identifier les deux pôles qui caractérisent la recherche dans les associations en Éducation à l'environnement: une recherche associative autonome centrée sur des questions contextualisées et associée à des réseaux d'échange de savoirs et de pratiques; une autre, en partenariat avec des institutions académiques. Or quel partenariat? Quels buts et quelles logiques? Ce sont là des questions auxquelles il est urgent de répondre car elles sont déterminantes pour l'avenir de l'Éducation à l'environnement. 


\section{BIBLIOGRAPHIE}

Bidou J.E. (2006), Un chercheur engagé ? Cahiers Scientifiques ACFAS n 104 , p. 203-219

Callon M., Lascoumes P. et Barthe Y. (2001), Agir dans un monde incertain. Essai sur la démocratie technique. Paris : Editions du Seuil.

Freire P. (1996). L'Éducation comme pratique de la liberté. Paris : Éditions W

Funtowicz S., Ravetz J-R. (1991). A New Scientific Methodology for Global Environmental Issue. Dans Costanza R. (ed.), The Ecological Economics (pp. 137-152). New York: Columbia University Press

Gingras Y. (2014), Les dérives de l'évaluation de la recherche. Du bon usage de la bibliométrie, Paris : Raisons d'agir

Lyet P. (2011), Traduction, transaction sociale et tiers intermédiaire dans les processus de collaboration de chercheurs et de praticiens dans le cadre de recherches-actions. Pensée plurielle 3

(28) $49-67$

Peyronie H. (2013), Le mouvement Freinet : du fondateur charismatique à l'intellectuel collectif. Caen : Presses universitaires de Caen

Richez J.C. (2010), Cinq contributions autour de l'éducation populaire. Paris : Institut national de la jeunesse et de l'éducation populaire. Consulté sur : http://www.injep.fr/sites/default/files/ documents/Richez_5contributions_educ-pop.pdf

Schön D. A. (1994) Le praticien réflexif. A la recherche du savoir caché dans l'agir professionnel. Montréal : Editions Logiques

\section{AUTEUR}

\section{JEAN-ÉTIENNE BIDOU}

Ifrée 